\title{
Design of Miniaturized On-Chip Bandpass Filters Using Inverting-Coupled Inductors in (Bi)-CMOS Technology
}

\author{
He Zhu, Member, IEEE, Xi Zhu, Member, IEEE, Yang Yang, Senior Member, IEEE, and Yichuang Sun, \\ Senior Member, IEEE
}

\begin{abstract}
In this work, a new type of miniaturized on-chip resonator using coupled-inductor structure is presented. The impact on resonances of the structure due to the use of noninverting- and inverting-coupled configuration is extensively investigated. It has been found that using the inverting-coupled structure, a stronger resonance can be generated, which is ideally suitable for device miniaturization. To fully understand the working mechanism of the resonator and use it effectively for bandpass filter (BPF) design, simplified $L C$ equivalent-circuit models and detailed theoretical analysis are provided. To further demonstrate the proposed concept is useful in practice, not only a $1^{\text {st }}$-order BPF, but also another two $2^{\text {nd }}$-order BPFs are designed and fabricated in a standard $0.13-\mu \mathrm{m}$ (Bi)-CMOS technology. All of them are designed to have a centre frequency around $15 \mathrm{GHz}$. Their physical dimensions are $0.13 \times 0.25 \mathrm{~mm}^{2}, 0.26 \times 0.25 \mathrm{~mm}^{2}$, $0.24 \times 0.22 \mathrm{~mm}^{2}$, respectively. Good agreements between simulation and measurement have been obtained, which verify that the presented design approach is suitable for miniaturized on-chip passive design.
\end{abstract}

Index Terms - Bandpass filters, inductively coupled resonator, inverting coupling, non-inverting coupling, on-chip resonator.

\section{INTRODUCTION}

Radio-frequency (RF) on-chip filter is a building block that can be found in many RF transceivers. One of the common design issues related to such filters is how to minimize their footprint. This is mainly due to the fact that passive components, such as spiral inductor (IND) and transmission line (TL), are inherently bulky and thus take a fairly large die area. To solve this issue without adversely affecting other performance, one of the possible techniques is to use active inductors to replace the passive ones, which have been extensively studied in the literature [1]-[2]. However, the performance of active inductors may be severely deteriorated, while the operation frequency goes above $10 \mathrm{GHz}$. Thus, research on miniaturized on-chip

Manuscript received XXX. This research is supported by the Australian Research Council DE160101032.

H. Zhu, X. Zhu and Y. Yang are with the School of Electrical and Data Engineering, Global Big Data Technology Centre (GBDTC), University of Technology Sydney, Ultimo, NSW 2007, Australia. Email: he.zhu@uts.edu.au; xi.zhu@uts.edu.au and yang.yang-1@uts.edu.au.

Y. Sun is with the School of Engineering and Computer Science, University of Hertfordshire, Hatfield AL10 9AB, U.K. E-mail: y.sun@ herts.ac.uk. passive components design to support microwave and millimetre-wave technologies is emerging [3]-[8]. Furthermore, another motivation behind device miniaturization is to reduce insertion loss of passive component. For operation frequency beyond $10 \mathrm{GHz}$, one of the major sources that increases insertion loss of passive component, especially for TLs and INDs, is the so-called ohmic loss, which is related to the overall length of components. If the overall length of TL or IND can be effectively reduced, the insertion loss of them could also be reduced. Several prior works have been presented in the literature for both silicon and III/V technologies [9]-[26]. The most classical method is to fold metal strip lines so that die area can be used more effectively [9]-[13]. One of the typical examples for this method is to use 3-D inductors for amplifier design [10]-[11]. However, simply folding a metal strip does not necessarily help to reduce its overall length. As a result, the insertion loss of such metal strip is unlikely to be reduced. To effectively reduce the insertion loss of a passive component, some other approaches have also been explored, such as slow-wave structure and co-planer waveguide [14]-[20]. However, a slow-wave structure is adopted for a design, the footprint of a passive component operating below $60 \mathrm{GHz}$ is still fairly large. To solve this issue, a hybrid approach that utilizes capacitive-loaded TLs or INDs has been extensively used in [21]-[26] Since metal-insulator-metal (MIM) layer is provided for the most RF-CMOS process and MIM capacitor can be implemented in a very compact way, a combination of using a relatively large capacitance with a small inductance to obtain the required resonant frequency is desirable.

In this paper, an interesting design approach is presented for miniaturized passive device implementation, especially for bandpass filters (BPFs). Two classical coupling structures, namely inverting and non-inverting coupling, are investigated. As will be shown, the inverting-coupled structure is very well suitable for BPF design with a reduced size, several BPF prototypes are developed with compact size and excellent performance. Thorough theoretical analysis is given to explore the insight characteristics of the presented approach and provide a guidance to design BPFs based on this concept. To prove the approach is feasible in practice, three BPFs are implemented and fabricated in a standard $0.13-\mu \mathrm{m}$ (Bi)-CMOS technology and good agreements between the EM simulated and measured results are achieved. 


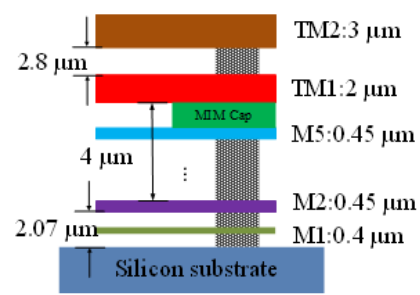

Fig. 1. Metal stack-up of the selected $0.13-\mu \mathrm{m}$ (Bi)-CMOS technology.

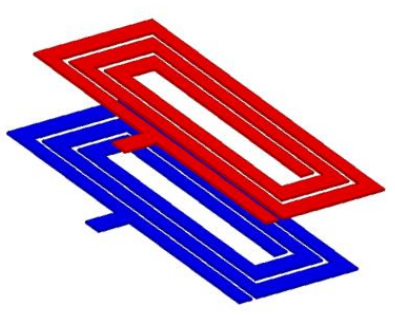

(a)

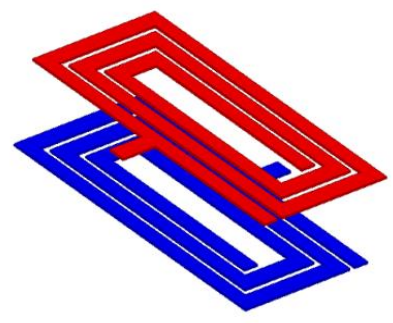

(b)
Fig. 2. Two types of resonators using coupled inductors: (a) non-inverting coupled type; (b) inverting coupled type.

The rest of this paper is organized as follows. In Section II, the inverting and non-inverting coupled structures are compared and discussed in terms of resonant poles and transmission zeros. In Section III, how to use the coupled structures for BPF design is described and design of a $1^{\text {st }}$ - and $2^{\text {nd }}$-order BPFs are given as examples. To further improve out-of-band suppression for the designed BPFs, a modified resonator and its application for BPF is further explored in Section IV. The measurement results are presented in Section V and the conclusion is finally drawn in Section VI.

\section{Design OF Miniaturized Resonator Using COUPLED-INDUCTOR STRUCTURE}

\section{A. Overview of the Resonators Design with Different Coupling Structures}

The back-end-of-line (BEOL) information is shown in Fig. 1. A standard $0.13-\mu \mathrm{m}(\mathrm{Bi})-\mathrm{CMOS} \mathrm{SiGe}$ technology is used in the design, which provides not only high-performance transistors (with $f_{\mathrm{t}}$ of $200 \mathrm{GHz}$ ), but also 7 metal layers (TM1, TM2 and M1 to M5) with aluminium as the thick top two metal layers (TM1 and TM2). The additional MIM layer is placed between the sixth and fifth layers (TM1 and M5). In addition, the height of the silicon substrate is $200 \mu \mathrm{m}$. The dielectric constant of $\mathrm{SiO}_{2}$ is 4.1 and the loss tangent is 0.01 . In this work, two types of self-coupled resonators based on non-inverting and inverting coupling types will be discussed.

In this section, both of non-inverting and inverting-coupled resonators are theoretically analysed. Both two resonators are built on the TM1 and TM2 layers. To understand the difference between two coupling structures, 3-D views of them are provided in Fig. 2. As can be seen, each structure consists of two spiral inductors, which are identical in terms of length and width. The difference between them is how the two spiral inductors are coupled with each other. As shown in Fig. 2(a), both the upper and lower spirals are implemented in a clock

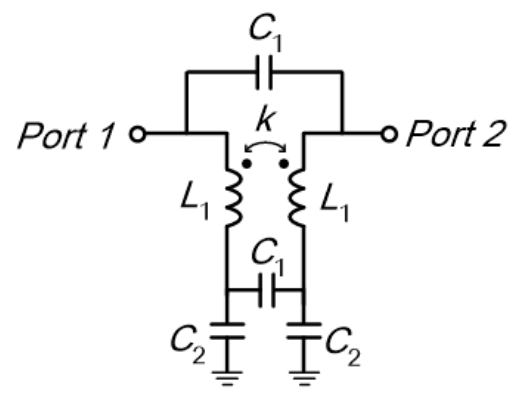

(a)

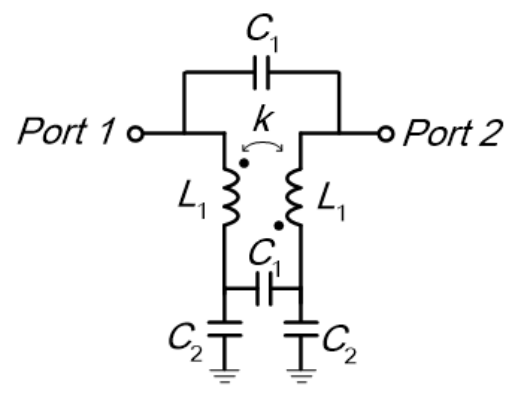

(b)

Fig. 3. Simplified $L C$-equivalent circuit models for the resonators in Fig. 2: (a) with non-inverting coupled one; (b) with inverting coupled one.

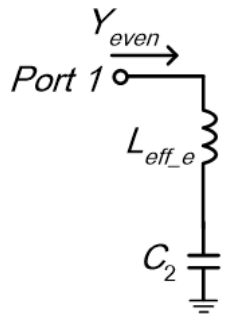

(a)

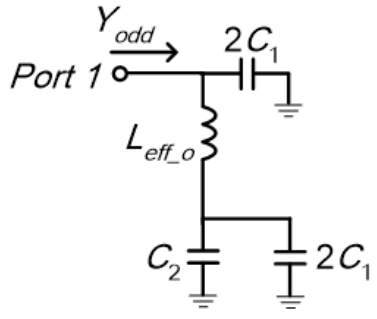

(b)
Fig. 4. Even- and odd-mode analysis of the $L C$-equivalent circuit: (a) evenmode, (b) odd-mode.

-wise rotating pattern, which is called non-inverting coupled structure. Unlike this structure, as illustrated in Fig. 2(b), the lower spiral is implemented in an anti-clockwise rotating pattern. It means that the lower spiral inductor is placed in an opposite orientation of the upper one, which is known as inverting-coupled structure. Using the inverting-coupled structure, a strong inductive coupling, also known as magnetic coupling, will occur between two spirals, which makes the structure operating as a resonator.

To further illustrate the insight of this structure, more investigation is given. It will be found that with the same dimensions, the inverting-coupled structure can produce a resonant mode at a relatively lower frequency than the non-inverting-coupled one. This means that to produce a resonance at certain frequency, the inverting-coupled resonator will occupy smaller size. As will be shown in the later sections, a series of BPFs will be designed and implemented using the inverting-coupled structure for miniaturized designs. To investigate the properties of the presented resonators, further analysis and discussions will be introduced in the following sub-sections. 


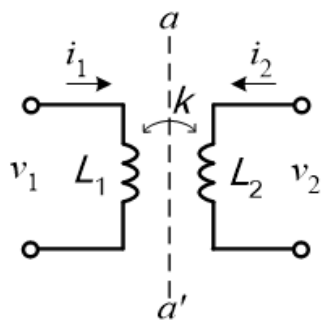

Fig. 5. A two-port network comprising two inductors including mutual coupling.

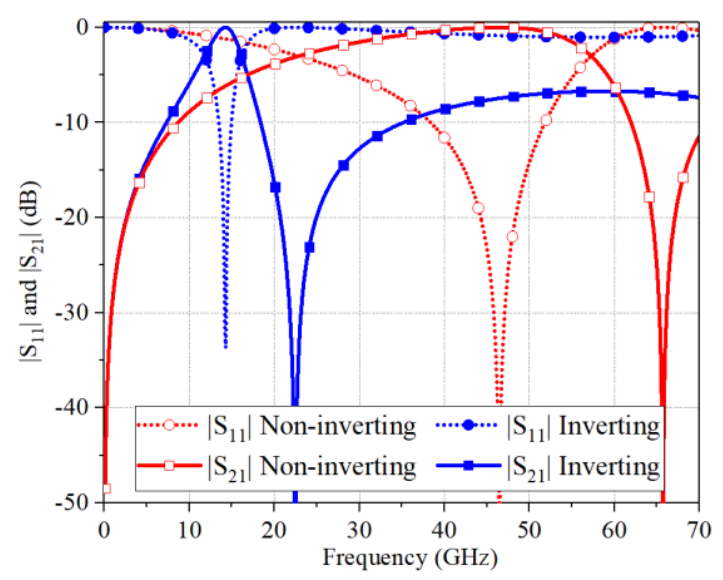

Fig. 6. Calculated $S$-parameters of two filters using inverting- and non-inverting- coupled structures. Related parameter values are: $L_{1}=1000 \mathrm{pH}$, $C_{1}=30 \mathrm{fF}, C_{2}=10 \mathrm{fF}$, and $k=-0.8$ (inverting coupling) and 0.8 (non-inverting coupling).

\section{B. Analysis of the Resonators}

The above-mentioned resonators can be modelled using simplified $L C$-equivalent circuits, which consist of lumped inductors and capacitors. In the analysis, since the resonator is regarded as lossless, no resistors are considered in the equivalent circuits. Corresponding to Fig. 2, the $L C$-equivalent circuits of two resonators are shown in Fig. 3, where Fig. 3(a) shows the equivalent circuit of the non-inverting-coupled resonator, while Fig. 3(b) shows the inverting-coupled one. Both two circuit models are composed of two inductors $L_{1}$ with inductive mutual coupling $M$, two mutual capacitances $C_{1}$ and two grounded capacitors $C_{2}$. The capacitance $C_{1}$ denotes the electrical coupling that exists between two metal lines. $C_{2}$ is the effective grounded capacitor existing between the metal lines and the ground. Regardless the type of the resonators, both of them can be analysed using even- and odd-mode analysis method. The mutual coupling between two coupled inductors can be evaluated using a coupling coefficient $k$, which is $k=M / L_{1}$ and $M$ represents the mutual inductance. For the case of non-inverting coupled structure, there is $0<k<1$; while for the case of inverting coupled one, there is $-1<k<0$.

The resonator can be bisected into two parts along the symmetric line: even-mode circuit and odd-mode circuit. For the even-mode circuit, the symmetric line can be regarded as a perfect magnetic conductor (PMC), and its equivalent circuit is shown in Fig. 4(a). It is a series circuit of an inductor and a capacitor. Due to the existence of mutual coupling $M$, the inductance in the even-mode circuit is denoted as $L_{e f f_{-} e}$.

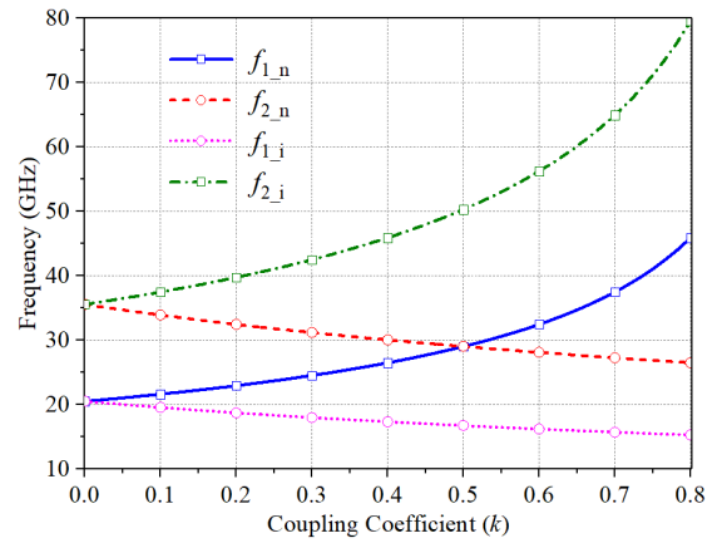

Fig. 7. Resonant mode distribution of $f_{1 \_n}, f_{2 \_n}, f_{1 \_i}$ and $f_{2 \_i}$ against $k$. Related parameter values are: $L_{1}=1000 \mathrm{pH}, C_{1}=20 \mathrm{fF}, C_{2}=20 \mathrm{fF}$.

Therefore, the input admittance of the even-mode circuit can be expressed as:

$$
Y_{\text {even }}=\frac{j \cdot \omega C_{2}}{1-\omega^{2} L_{\text {eff_e }} \cdot C_{2}}
$$

For the odd-mode circuit, the symmetric line can be regarded as a perfect electric conductor (PEC), and its equivalent circuit is shown in Fig. 4(b). It is composed of grounded capacitors and a series circuit of an inductor and two paralleled capacitors. Similar to the even-mode case, due to the existence of mutual coupling $M$, the inductance in the odd-mode circuit can be regarded as $L_{e f f_{-} o}$. Therefore, the input admittance of the odd-mode circuit can be expressed as:

$$
Y_{\text {odd }}=\frac{j \cdot \omega\left(2 C_{1}+C_{2}\right)}{1-\omega^{2} L_{\text {eff } \_o} \cdot\left(2 C_{1}+C_{2}\right)}+j \cdot 2 \omega C_{1}
$$

To determine the value of input admittance $Y_{\text {even }}$ and $Y_{\text {odd }}$, the effective inductances of $L_{e f f_{-} e}$ and $L_{e f f_{-} o}$ must be solved. Fig. 4 displays a two-port network comprising of only two inductors with mutual inductive coupling $M$, which is equal to $k \sqrt{L_{1} L_{2}}$. Assuming that $L_{1}=L_{2}=L$, the value of $M$ is ranged between 0 and $L$. The voltage and current distribution is also marked in Fig. 4. For any inductor $L$ itself without mutual coupling, there is $v_{1}=i_{1} \cdot X_{l}=i_{1} \cdot j \omega L$, so

$$
L=\frac{v_{1}}{j \omega \cdot i_{1}}
$$

When the mutual coupling $M$ is considered, the effective inductance is:

$$
L_{e f f}=\frac{v_{1}}{j \omega \cdot i_{1}}=\frac{j \omega L \cdot i_{1}+j \omega M \cdot i_{2}}{j \omega \cdot i_{1}}=L+M \frac{i_{2}}{i_{1}}
$$

For the even-mode circuit, the symmetric line $a-a$ ' is regarded as a PMC. According to the mirror rule, the image current along the PMC has the same direction and magnitude of the original one; hence, $i_{2}=i_{1}$. Therefore, the effective inductance $L_{e f f_{-} e}$ in the even-mode circuit is

$$
L_{e f f_{e}}=L+M
$$

For the odd-mode circuit, the symmetric line $a-a$ ' is regarded as a PEC. According to the mirror rule, the image 


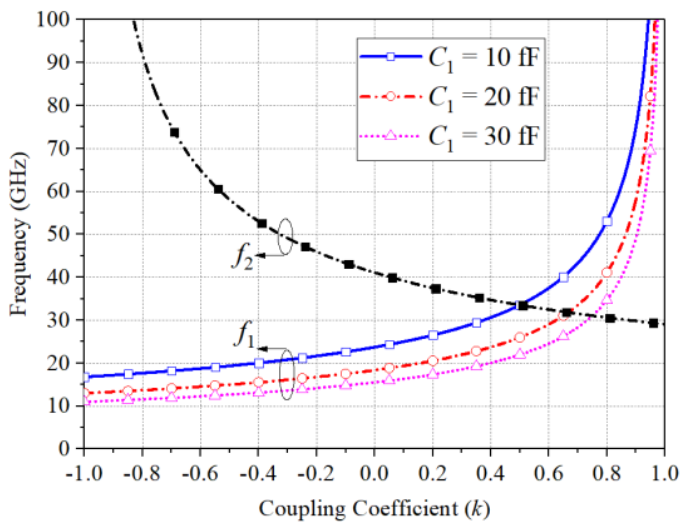

(a)

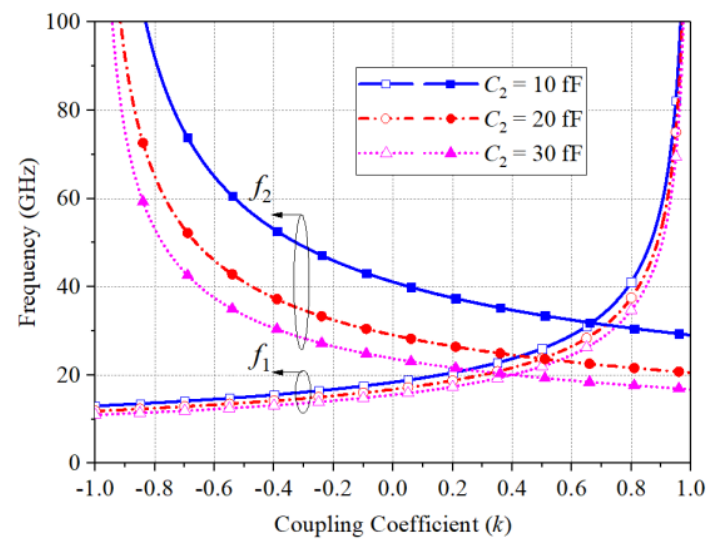

(b)

Fig. 8. The values of resonant poles against coupling coefficient $k$ when different values of $C_{1}$ and $C_{2}$ are chosen: (a) $C_{1}$ is varied from 10 to $30 \mathrm{fF}$, (b) $C_{2}$ is varied from 10 to $30 \mathrm{fF}$.

current along the PEC has the same magnitude and opposite direction compared with the original one; hence, in this case $i_{2}=-i_{1}$, and thus the odd-mode effective inductance $L_{\text {eff_o }}$ is

$$
L_{e f f_{-} o}=L-M
$$

From (5) and (6), it is obviously seen that the value of $L_{e f f_{-} e}$ will increase with $M$ and its value is ranged from $L$ and $2 L$; while $L_{e f f_{-} o}$ will decrease with $M$ and its value is ranged from 0 and $L$. Using (5) and (6), the even- and odd-mode input admittance can be rewritten as:

$$
\begin{gathered}
Y_{\text {even }}=\frac{j \cdot \omega C_{2}}{1-\omega^{2}\left(L_{1}+M\right) C_{2}} \\
Y_{\text {odd }}=\frac{j \cdot \omega\left(2 C_{1}+C_{2}\right)}{1-\omega^{2}\left(L_{1}-M\right)\left(2 C_{1}+C_{2}\right)}+j \cdot 2 \omega C_{1}
\end{gathered}
$$

The resonances occur when the input admittance of the even- and odd-mode circuit approaches infinity. Solving $Y_{\text {even }}=\infty$ and $Y_{\text {odd }}=\infty$, it is possible to find the locations of two resonant frequencies of the resonator:

$$
\begin{aligned}
& f_{1}=\frac{1}{2 \pi \sqrt{\left(L_{1}-M\right) \cdot\left(2 C_{1}+C_{2}\right)}} \\
& f_{2}=\frac{1}{2 \pi \sqrt{\left(L_{1}+M\right) \cdot C_{2}}}
\end{aligned}
$$

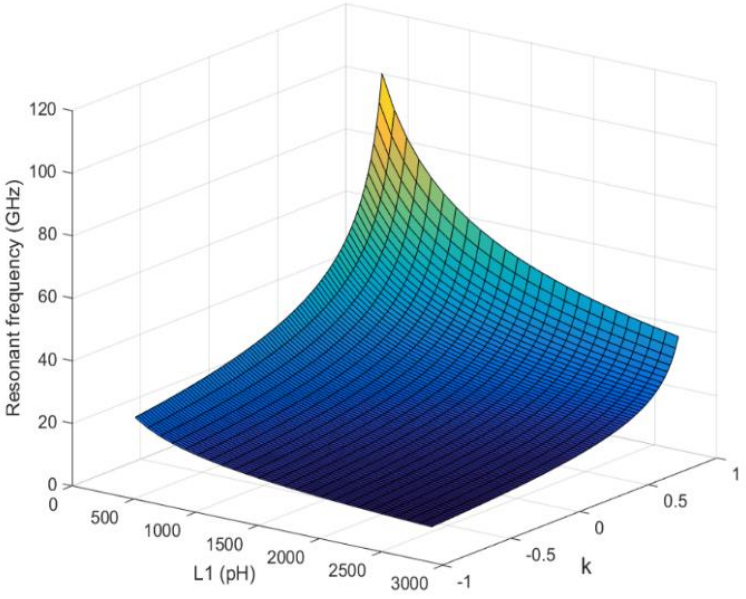

Fig. 9. 3D mapping of resonant frequency $f_{1}$ against $k$ and $L_{1}$.

When the resonator is directly connected with $\mathrm{I} / \mathrm{O}$ ports, the resonator will perform a filtering characteristic. Using the expressions of the even- and odd-mode input admittance, it is possible to calculate the scattering parameters ( $S$-parameters) of the filter, which can be expressed as [27]:

$$
\begin{aligned}
& S_{21}=\frac{Y_{o}\left(Y_{\text {odd }}-Y_{\text {even }}\right)}{\left(Y_{o}+Y_{\text {odd }}\right)\left(Y_{o}+Y_{\text {even }}\right)} \\
& S_{11}=\frac{Y_{o}^{2}-Y_{\text {even }} Y_{\text {odd }}}{\left(Y_{o}+Y_{\text {odd }}\right)\left(Y_{o}+Y_{\text {even }}\right)}
\end{aligned}
$$

Two different circuits with inverting- and non-invertingcoupled resonators are calculated and compared. Fig. 6 depicts the $S$-paramters of two BPFs based on these two different resonators, respectively. It is seen that when the coupling coefficients are the same in magnitude ( $k$ equals to -0.8 for inverting coupling and 0.8 non-inverting coupling) with opposite signs, the $S$-paraemters are totally different. This is because different types of coupling result in different resonant frequencies and transmission zeros of the filtering responses. To find out the intrisinc machnism, it is important to investigate the resonant modes and transmission zeros that are produced by the resonators.

\section{Resonant Modes of the Resonators}

As observed from Fig. 6, the resonance of the invertingcoupled structure occurs at about $14 \mathrm{GHz}$, while the resonance of the non-inverting-coupld one is at around $45 \mathrm{GHz}$, which is almost three times higher than the inverting-coupled case. This can be explained by (9) and (10). For the non-inverting-coupled case, the mutual inductance $M$ is positive, and two resonant frequenies are written as $f_{1 \_n}$ and $f_{2 \_n}$; on the other hand, for the case of inverting coupling, the mutual inductance $M$ is negative, and two resonate frequencies are written as $f_{1_{-} i}$ and $f_{2_{-} i}$. When the same amount of coupling inductance is considered in two cases, the resonant poles of $f_{1 \_n}, f_{2 \_n}, f_{1 \_i}$ and $f_{2_{-} i}$ are located at different positions on the spectrum. As an example, when $L_{1}$ is selected as $1000 \mathrm{pH}, C_{1}=20 \mathrm{fF}$ and $C_{2}=20$ $\mathrm{fF}$, the resonant mode distribution of the resonator is displayed in Fig. 7. When $0<|k|<0.5$, there is $f_{1 \_i}<f_{1 \_n}<f_{2 \_n}<$ 


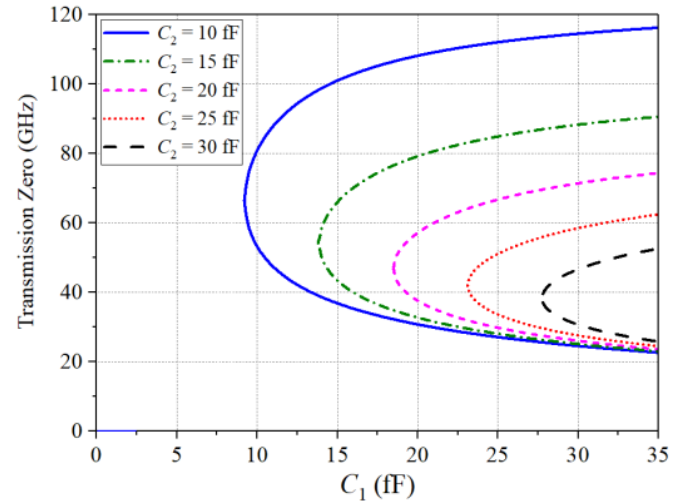

(a)

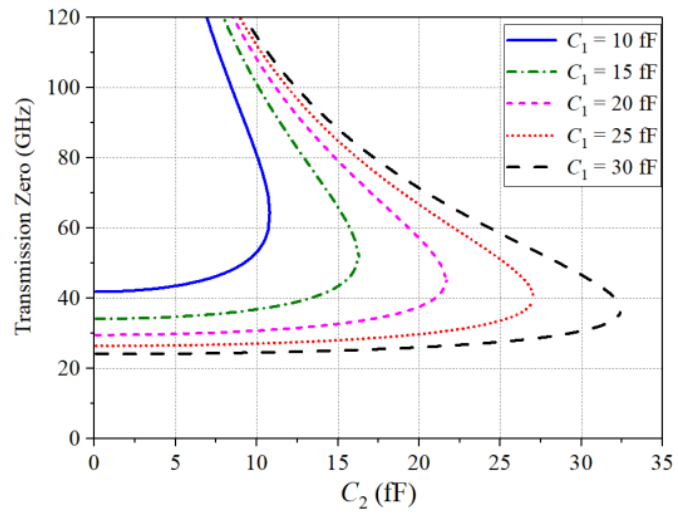

(b)

Fig. 10. The values of transmission zeros (a) varying against $C_{1}$ when $C_{2}$ is fixed; (b) varying against $C_{2}$ when $C_{1}$ is fixed.

$f_{2 \_}$; while when $0.5<|k|<1$, there is $f_{1 \_i}<f_{2_{-} n}<f_{1 \_n}<$ $f_{2-i}$. In both cases $(0<|k|<1)$, it is found $f_{1-i}$ is always located at the lowest frequency. The curves in Fig. 7 may vary when different values of $C_{1}$ and $C_{2}$ are chosen, but the relation of resonant poles is similar. This means that if the two resonators have same dimensions, the inverting-coupled one is likely to get its resonate pole located at the lower frequency. Besides, this phenomenon also explains why the calculated $\left|S_{21}\right|$ of the inverting-coupled case is located at a much lower frequency than the non-inverting coupled one, as is indicated in Fig. 6. It is obvious that the inverting-coupled one can produce a resonant pole at much lower frequency, which is favourable to be used in designing miniaturized BPF.

Fig. 8 shows the relation between $k$ and $f_{1}, f_{2}$ with different values of $C_{1}$ and $C_{2}$. When $|k|$ is fixed, a smaller $C_{1}$ will result in a higher $f_{1}$, as seen from (9); on the other hand, the variance of $C_{1}$ does not affect $f_{2}$ because $f_{2}$ has nothing to do with $C_{1}$ as seen from (10). Similarly, a smaller $C_{2}$ will result in a higher $f_{1}$ and $f_{2}$, because $C_{2}$ contributes to the denominators of both $f_{1}$ and $f_{2}$ in (9) and (10). Since the capacitances of $C_{1}$ and $C_{2}$ are relatively small and constant (typically around 10-30 fF), the main affecting factor of the resonant poles to $f_{1}$ and $f_{2}$ are $L_{1}$ and $k$. Fig. 8 shows a 3-D mapping relation of resonant $f_{1}$ against $L_{1}$ and $k$. It is observed that a lower value of $f_{1}$ occurs at a larger value of $L_{1}$ and a larger value of $|k|$ when $k$ is negative. In this design, the first resonant mode of the resonator $f_{1}$ under inverting coupled structure (as written as $f_{1_{-} i}$ ) will be

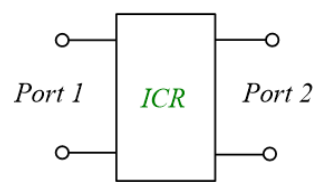

(a)

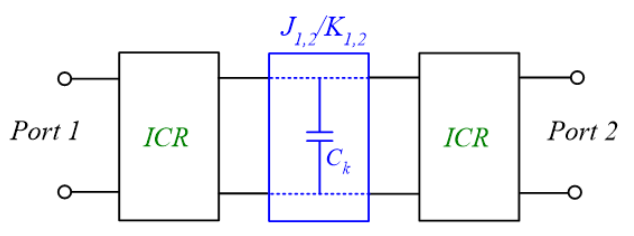

(b)

Fig. 11. Configuration of (a) a conventional $1^{\text {st }}$-order BPF; (b) a conventional $2^{\text {nd }}$-order BPF. Note: ICR stands for inverting-coupled resonator.

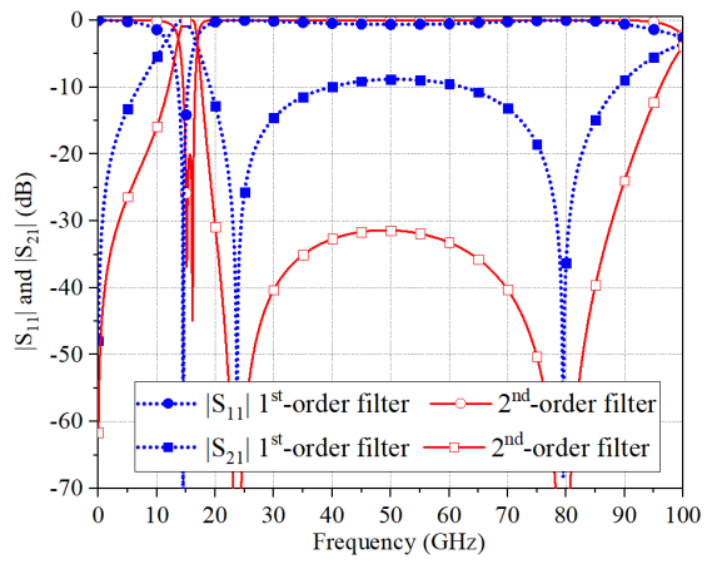

Fig. 12. S-parameters of the designed BPFs using inverting-coupled structure.

utilized as the resonant pole for design of BPFs in the following sections of this paper.

\section{Transmission Zeros}

Apart from the resonant modes, the inverting-coupled resonator can also generate multiple transmission zeros, which can be used to improve out-of-band suppression. The transmission zeros occur when the even-mode impedance and odd-mode impedance are equal to each other, which is $Y_{\text {even }}=$ $Y_{\text {odd }}$. In this case, from (7) and (8) it is forced to have

$$
\frac{j \cdot \omega C_{2}}{1-\omega^{2}\left(L_{1}+k \cdot L_{1}\right) C_{2}}=\frac{j \cdot \omega\left(2 C_{1}+C_{2}\right)}{1-\omega^{2}\left(L_{1}-k \cdot L_{1}\right)\left(2 C_{1}+C_{2}\right)}+j \cdot 2 \omega C_{1}
$$

By solving (12), two solutions to $\omega$ can be derived, which correspond to the transmission zeros $\omega_{t z 1}$ and $\omega_{t z 2}$ that can be expressed as:

$$
\begin{aligned}
& \omega_{t z 1}=\sqrt{\frac{2 C_{1}^{2}+2 C_{1} C_{2}-2 C_{1}^{2} k+C_{2}^{2} k+2 C_{1} C_{2} k-\sqrt{P}}{2 Q}} \\
& \omega_{t z 2}=\sqrt{\frac{2 C_{1}^{2}+2 C_{1} C_{2}-2 C_{1}^{2} k+C_{2}^{2} k+2 C_{1} C_{2} k+\sqrt{P}}{2 Q}}
\end{aligned}
$$

where

$$
\begin{gathered}
P=4 C_{1}^{4} k^{2}-8 C_{1}^{4} k+4 C_{1}^{4}+8 C_{1}^{3} C_{2} k^{2}-8 C_{1}^{3} C_{2}+8 C_{1}^{2} C_{2}^{2} k^{2} \\
+12 C_{1}^{2} C_{2}^{2} k-4 C_{1}^{2} C_{2}^{2}+4 C_{1} C_{2}^{3} k^{2}+4 C_{1} C_{2}^{3} k+C_{2}^{4} k^{2} \\
Q=-2 L_{1} C_{1}^{2} C_{2} k^{2}+2 L_{1} C_{1}^{2} C_{2}-L_{1} C_{1} C_{2}^{2} k^{2}+L_{1} C_{1} C_{2}^{2}
\end{gathered}
$$




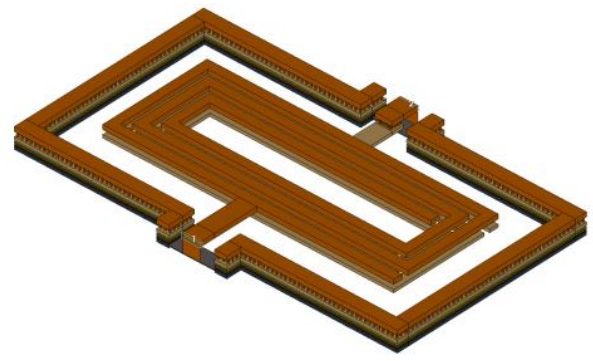

(a)

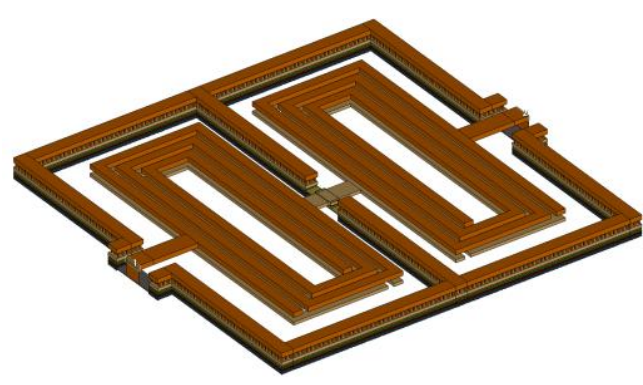

(b)

Fig. 13. 3-D views of the designed BPFs, (a) $1^{\text {st }}$-order one, (b) $2^{\text {nd }}$-order one.

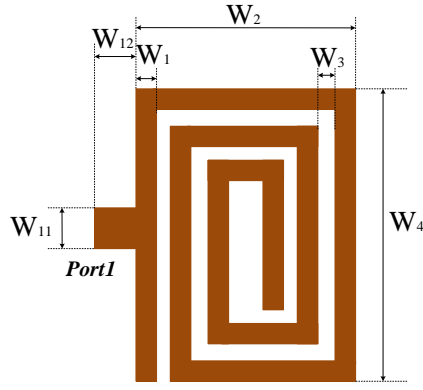

(a)

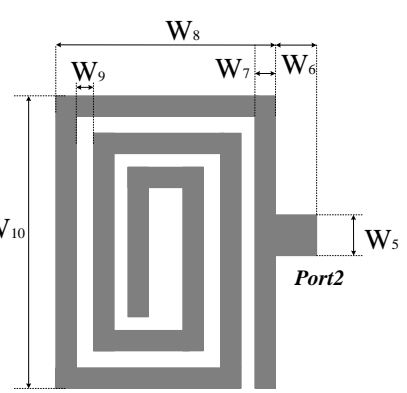

(b)
Fig. 14. Layout of the $1^{\text {st }}$-order BPF: (a) upper layer spiral, and (b) lower layer spiral. Note: $\mathrm{W}_{1}=6 \mu \mathrm{m}, \mathrm{W}_{2}=74 \mu \mathrm{m}, \mathrm{W}_{3}=4 \mu \mathrm{m}, \mathrm{W}_{4}=190 \mu \mathrm{m}, \mathrm{W}_{5}=12 \mu \mathrm{m}$, $\mathrm{W}_{6}=12 \mu \mathrm{m}, \mathrm{W}_{7}=6 \mu \mathrm{m}, \mathrm{W}_{8}=74 \mu \mathrm{m}, \mathrm{W}_{9}=4 \mu \mathrm{m}, \mathrm{W}_{10}=190 \mu \mathrm{m}, \mathrm{W}_{11}=12 \mu \mathrm{m}$, and $\mathrm{W}_{12}=12 \mu \mathrm{m}$.

The expressions of $\omega_{t z 1}$ and $\omega_{t z 2}$ are complicated and it is difficult to identify the effect of $L_{1}, C_{1}$ and $C_{2}$ on the zeros. However, by observing (13) and (14), one can find that $L_{1}$ is always on the denominators of $\omega_{t z 1}$ and $\omega_{t z 2}$, which means the positions of transmission zeros are inversely proportional to $L_{1}$. Moreover, to investigate the effect of $C_{1}$ and $C_{2}$ on $\omega_{t z 1}$ and $\omega_{t z 2}$, multiple cases with different values of $C_{1}$ and $C_{2}$ against the locations of transmission zeros, $f_{t z 1}$ and $f_{t z 2}$, (which are equal to $\omega_{t z 1}$ and $\omega_{t z 2}$ divided by $2 \pi$ ), are shown in Fig. 10. When $C_{2}$ is fixed and $C_{1}$ increases from 10 to $30 \mathrm{fF}$, two transmission zeros will move closer to each other. When $C_{1}$ is smaller than a certain value, two transmission zeros will merge and disappear. This means that under such circumstance, equation (12) has no solutions. On the other hand, when $C_{1}$ is fixed and $C_{2}$ increases from 10 to $30 \mathrm{fF}, f_{t z 1}$ will move to lower frequencies, while $f_{t z 2}$ will move further higher. When $C_{2}$ is higher than a certain value, $f_{t z 1}$ and $f_{t z 2}$ will disappear as well. Therefore, it is quite important to select appropriate values for $C_{1}$ and $C_{2}$ to guarantee the transmission zeros are existing at the upper-stopband during the procedure of BPF design.

\section{DESIGN OF $1^{\mathrm{ST}}$-ORDER AND $2^{\mathrm{ND}}$-ORDER BPFS}

\section{A. $1^{\text {st }}$-Order BPF}

The presented inverting-coupled resonator has been proved to be able to produce multiple resonances and transmission zeros due to the inductive coupling between two metal layers. The resonances and transmission zeros can be easily controlled. Therefore, this type of resonator is favoured for BPF designs. As one knows, if the resonator is directly taped with two feeds, as indicated in Fig. 11(a), it is possible to build a $1^{\text {st }}$-order filter. Since the resonator can provide a transmission zero at DC, this $1^{\text {st }}$-order filter is a bandpass type. Apparently, the external quality factor is fixed, which has been pre-determined by the dimensions of the resonator. The external quality factor can be expressed as:

$$
\begin{gathered}
Q_{e x}=\frac{2 Y_{o} g_{o} g_{1}}{b_{r}} \\
b_{r}=\frac{\omega_{o} \cdot \partial \operatorname{Im}\left[Y_{\text {even }}\left(\omega_{o}\right)+Y_{o d d}\left(\omega_{o}\right)\right]}{4 \cdot \partial \omega}
\end{gathered}
$$

where $g_{o}$ and $g_{1}$ are the basic element value of a conventional $n$-stage lowpass filter prototype, and $b_{r}$ is the the susceptance slope parameter of the resonator [27]. $Y_{\text {even }}$ and $Y_{\text {odd }}$ can be calculated from (7) and (8). In addition, the fractional bandwidth (FBW) of the passband is also fixed, due to the relation between the FBW and $Q_{e x}$ :

$$
\begin{aligned}
& F B W=\frac{g_{0} g_{1}}{Q_{e x}}=\frac{b_{r} Y_{0}}{J_{0,1}^{2}} \\
& J_{0,1}=\sqrt{\frac{b_{r} Y_{0}}{Q_{e x}}}=\sqrt{\frac{b_{r} F B W Y_{0}}{g_{0} g_{1}}}
\end{aligned}
$$

where $J_{0,1}$ refers to the value of the admittance inverter at the input/output port. Since no extra feeding network is used in Fig. $11(\mathrm{a}), J_{0,1}$ is also a fixed value in this design. To adjust the in-band ripple and out-of-band performance, a higher-order filter needs to be developed.

\section{B. $2^{\text {nd }}$-Order BPF}

Fig. 11(b) shows the configuration of a $2^{\text {nd }}$-order BPF which is also based on the presented inverting-coupled resonator. This $2^{\text {nd }}$-order BPF is composed of two identical resonators (previously used for $1^{\text {st }}$-order BPF implementation) and a shunted capacitor $C_{k}$ in the middle, which acts like a $J / K$ inverter to get the specified in-band coupling coefficient. The coupling coefficient can be expressed as:

$$
k_{1,2}=\frac{F B W}{\sqrt{g_{1} g_{2}}}=\frac{J_{1,2}}{b_{r}}
$$

where

$$
J_{1,2}=F B W \sqrt{\frac{b_{r}^{2}}{g_{1} g_{2}}}
$$

$J_{1,2}$ is the value of admittance inverter between two resonators, and it is realized by the shunted capacitor $C_{k}$. Therefore, the value of $C_{k}$ can be found using the following formula: 


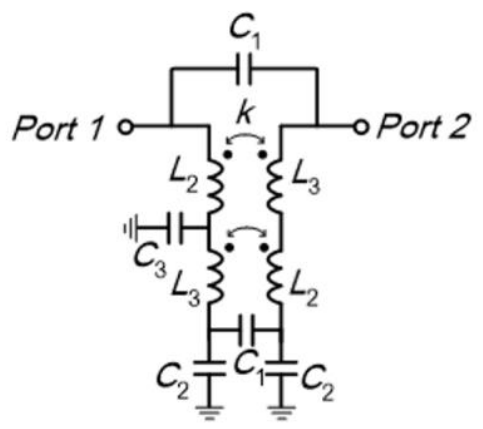

Fig. 15. Simplified $L C$-equivalent circuit of the modified inverting-coupled resonator.

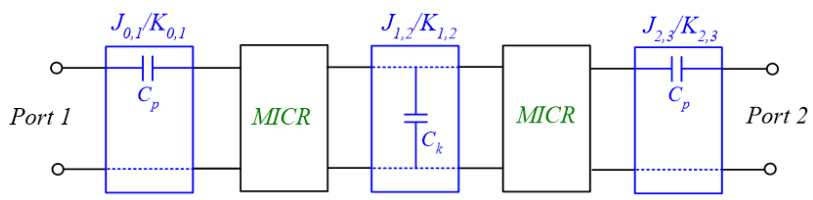

Fig. 16. Configuration of a $2^{\text {nd }}$-order BPF with controllable external $Q$-factor.

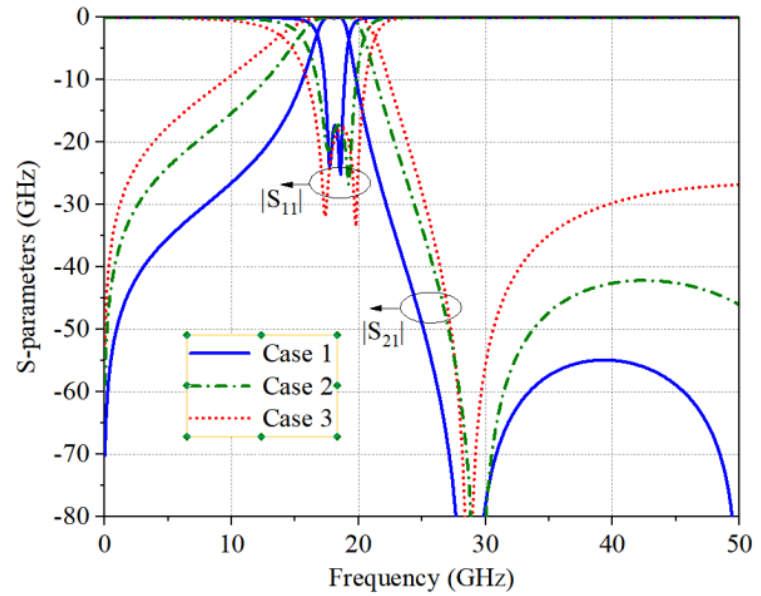

Fig. 17. S-parameters of three $2^{\text {nd }}$-order BPFs using MICR with different bandwidths.

$$
C_{k}=\frac{J_{1,2}}{2 \pi f_{o}}
$$

As indicated in (12), to provide a suitable coupling between two resonators, the value of $C_{k}$ should be determined according to $J_{1,2}$, which should be found based on the selection of $F B W$ and $b_{r}$.

The S-parameters of the $1^{\text {st }}$ - and $2^{\text {nd }}$-order BPFs are plotted in Fig. 12. Both of them have the same resonant poles and transmission zeros, which is because the same resonator is used. However, compared with the $1^{\text {st }}$-order BPF, the $2^{\text {nd }}$-order one has one more pole within the passband. Consequently, it provides a sharper selectivity and a better out-of-band performance. The $2^{\text {nd }}$-order BPF also has a more constant and flat in-band ripple compared with the $1^{\text {st }}$-order one. Although a good filtering response is achieved, the $2^{\text {nd }}-$ order BPF also has a fixed bandwidth, which is a major drawback and should be improved. Finally, the 3-D view of the $1^{\text {st }}$ - and $2^{\text {nd }}-$ order BPFs is given in Fig. 13 and the physical dimensions used for electromagnetic (EM) simulation are summarized in Fig. 14.

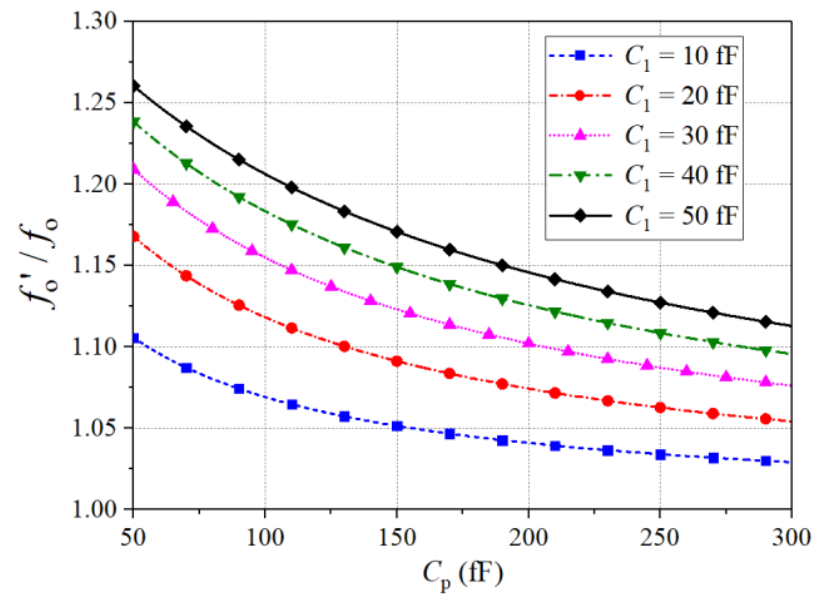

Fig. 18. The frequency ratio of $f_{o}^{\prime} / f_{o}$ against $C_{p}$ when $C_{1}$ varies from $10 \mathrm{fF}$ to $50 \mathrm{fF}$.

\section{DESIGN OF MODIFIED $2^{\mathrm{ND}}$-ORDER BPF WITH BANDWIDTH CONTROL AND IMPROVED UPPER-STOPBAND SUPPRESSION}

\section{A. Modified Inverting-Coupled Resonator}

In Section III, a $2^{\text {nd }}$-order filter is designed based on two inverting-coupled resonators. By controlling the equivalent inductance of metal lines and the inter-stage capacitance between them, it is possible to allocate the resonance at the bandpass frequency and the harmonic resonance at the upper stopband. Meanwhile, two transmission zeros are generated which helps to enhance the passband selectivity and stopband suppression. Based on the existing design, a modified resonator which is called modified inverting-coupled resonator is proposed here, as shown in Fig. 15. Compared with the previously presented resonator, this one has an extra shunted capacitor $C_{3}$, which is located at one of the metal lines of the resonator, splitting $L_{1}$ into two parts, $L_{2}$ and $L_{3}$. When $C_{3}$ is in the middle point of the metal line, there is $L_{2}=L_{3}=L_{1} / 2$. In this case, two spiral inductors become asymmetric, which would contribute to an additional transmission zero in the upper stopband of the filter.

\section{B. Modified 2 $2^{\text {nd }}$-Order BPF with Controllable Bandwidth}

Based on the modified resonator, an improved $2^{\text {nd }}-$ order BPF is built using the topology shown in Fig. 16. Compared with the initial $2^{\text {nd }}$-order BPF in Fig. 11(b), the modified design includes two cascaded capacitors $C_{p}$ at the input/output feeds. The cascaded $C_{p}$ plays the role of a $\mathrm{J} / \mathrm{K}$ inverter, which can control the external quality factor and $F B W$. The bandwidth of the filter $F B W$ can be expressed as:

$$
F B W=\frac{\left(J_{01}^{2} / Y_{o}\right) g_{0} g_{1}}{\frac{\omega_{o} \cdot \partial \operatorname{Im}\left[Y_{\text {even }}\left(\omega_{o}\right)+Y_{\text {odd }}\left(\omega_{o}\right)\right]}{4 \cdot \partial \omega}}
$$

Therefore, to get a certain value of FBW, the required value of the admittance inverter $J_{0,1}$ and the capacitance $C_{p}$ at the input/output port can be found to be:

$$
J_{0,1}=\sqrt{\frac{\omega_{o} \cdot F B W \cdot Y_{o} \cdot \partial \operatorname{Im}\left[Y_{\text {even }}\left(\omega_{o}\right)+Y_{\text {odd }}\left(\omega_{o}\right)\right]}{4 \cdot g_{0} g_{1} \cdot \partial \omega}}
$$




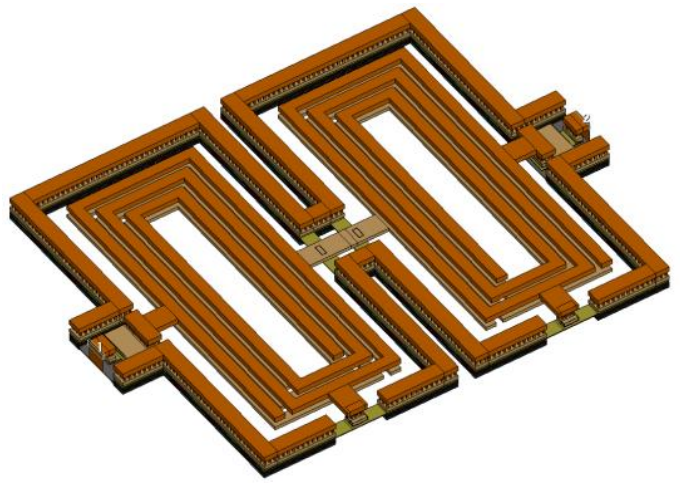

(a)

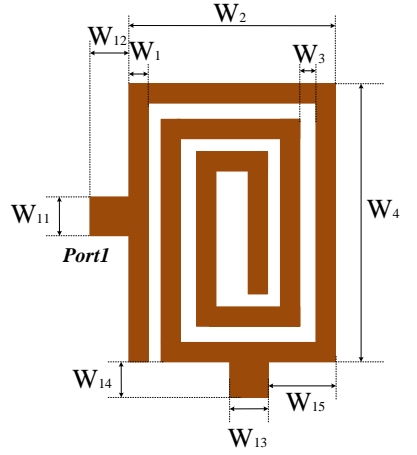

(b)

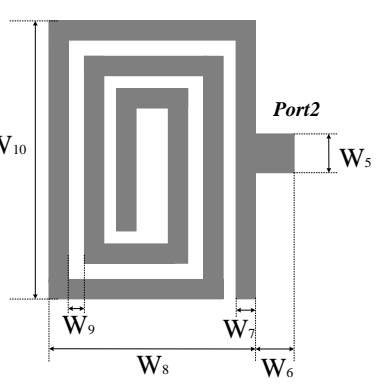

(c)
Fig. 19. Layout of the modified $2^{\text {nd }}$-order BPF: (a) 3-D view of the modified $2^{\text {nd }}$-order BPF, (b) upper spiral layer; and (c) lower spiral layer. Note: $\mathrm{W}_{1}=6$ $\mu \mathrm{m}, \mathrm{W}_{2}=74 \mu \mathrm{m}, \mathrm{W}_{3}=4 \mu \mathrm{m}, \mathrm{W}_{4}=180 \mu \mathrm{m}, \mathrm{W}_{5}=12 \mu \mathrm{m}, \mathrm{W}_{6}=12 \mu \mathrm{m}, \mathrm{W}_{7}=6$ $\mu \mathrm{m}, \mathrm{W}_{8}=74 \mu \mathrm{m}, \mathrm{W}_{9}=4 \mu \mathrm{m}, \mathrm{W}_{10}=180 \mu \mathrm{m}, \mathrm{W}_{11}=12 \mu \mathrm{m}, \mathrm{W}_{12}=12 \mu \mathrm{m}, \mathrm{W}_{13}$ $=12 \mu \mathrm{m}$ and $\mathrm{W}_{14}=12 \mu \mathrm{m}$.

$$
C_{p}=\frac{J_{0,1}}{2 \pi f_{o} \sqrt{1-\left(J_{0,1} / Y_{o}\right)^{2}}}
$$

When the resonator is fixed, the susceptance slope parameter $b_{r}$ is also fixed. Thus, the FBW is inverse proportional to $J_{0,1}^{2}$, which means a smaller value of $J_{0,1}$ will result in a larger bandwidth of the BPF. Based on this relation, the bandwidth of the passband can be simply controlled by changing the value of $C_{p}$, which is closely related to $J_{0,1}$. Fig. 17 shows the S- parameters of three cases with different FBW, while the same resonator is used. It is clearly seen that three bandpass filtering responses are realized with exactly the same center frequency and transmission zero, but different bandwidth. When $C_{p}$ is varied from $5 \mathrm{fF}$ to $100 \mathrm{fF}$, the FBW can be tuned from $10 \%$ to $30 \%$. Meanwhile, by slightly adjusting the value of $C_{k}$, one can make the in-band ripple at an optimal level.

\section{Frequency Shift in the Modified $2^{\text {nd }}$-Order BPF}

Due to the existence of $C_{p}$, the resonant frequency will shift to a higher frequency. This is because $C_{p}$ also contributes to a part of the resonator in the modified $2^{\text {nd }}-$ order bandpass filter. To investigate the frequency shift range, the equivalent evenand odd-mode admittance including the effect of $C_{p}$ should be modified as:

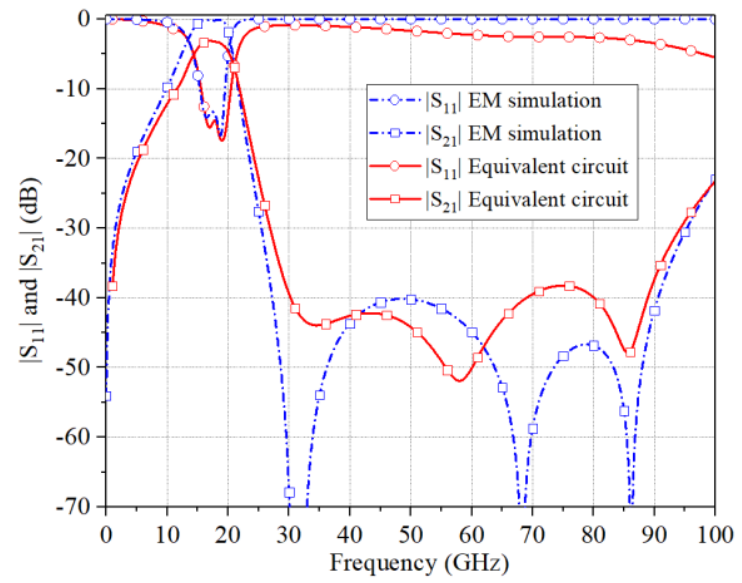

Fig. 20. Comparisons between the S-parameters of the modified $2^{\text {nd }}$-order BPF obtained from EM simulation and simplified circuit model.

$$
\begin{gathered}
Y_{\text {even }}^{\prime}=\frac{j \omega C_{p} \cdot Y_{\text {even }}}{j \omega C_{p}+Y_{\text {even }}} \\
Y_{\text {odd }}^{\prime}=\frac{j \omega C_{p} \cdot Y_{\text {odd }}}{j \omega C_{p}+Y_{\text {odd }}}
\end{gathered}
$$

Since the resonance in the passband occurs under the conditions of $Y_{\text {odd }}^{\prime}=\infty$, the resonant frequency with the effect of $C_{p}$ can be calculated as:

$$
f_{o}^{\prime}=\frac{1}{2 \pi} \sqrt{\frac{4 C_{1}+C_{2}+C_{p}}{\left(2 C_{1}+C_{p}\right) \cdot\left(L_{1}-M\right) \cdot\left(2 C_{1}+C_{2}\right)}}
$$

Compared with the original resonant frequency $f_{o}$ given in (9), the resonant frequency $f_{o}^{\prime}$ in the modified $2^{\text {nd }}$-order BPF has a factor of $q$, where

$$
q=\sqrt{\frac{4 C_{1}+C_{2}+C_{p}}{2 C_{1}+C_{p}}}
$$

In other words, $f_{o}^{\prime}=f_{o} \cdot q$. It is obvious that $q>1$, which means the original resonant frequency will definitely move to a higher frequency due to the existence of $C_{p}$. Since the value of $C_{p}$ is much larger than $C_{1}$ and $C_{2}$, the value of $q$ is just a little bit larger than 1. Fig. 18 shows the relation between the ratio of $f_{o}^{\prime} / f_{o}$ against $C_{p}$ when $C_{1}$ varies from $10 \mathrm{fF}$ to $50 \mathrm{fF}$. It is clearly seen that the ratio of $f_{o}^{\prime} / f_{o}$ moves towards 1 when $C_{p}$ increases, and a larger $C_{1}$ will lead to a more frequency shift of $f_{o}^{\prime}$ from $f_{o}$. This frequency shift can be compensated by slightly tuning the parameters of the BPF, such as $L_{1}$ and $C_{k}$, to adjust the resonant frequency and coupling coefficient back to the original status.

Fig. 19 shows the 3-D view as well as the layout of the modified $2^{\text {nd }}$-order BPF. Moreover, to prove that the presented simplified $L C$-equivalent circuit model is sufficiently accurate for predicting the characteristic of the design, EM simulated results are compared with those from the circuit model, which are shown in Fig. 19. The following values are chosen for the relevant parameters in the equivalent circuit: $L_{2}=450 \mathrm{pH}, L_{3}=$ $100 \mathrm{pH}, C_{1}=35 \mathrm{fF}, C_{2}=25 \mathrm{fF}, C_{p}=250 \mathrm{fF}, C_{k}=100 \mathrm{fF}$, and $k$ $=-0.9$. It is clearly seen that three transmission zeros are located at the upper-stopband of the filter, which greatly improves the harmonic suppression capability of the filter. 


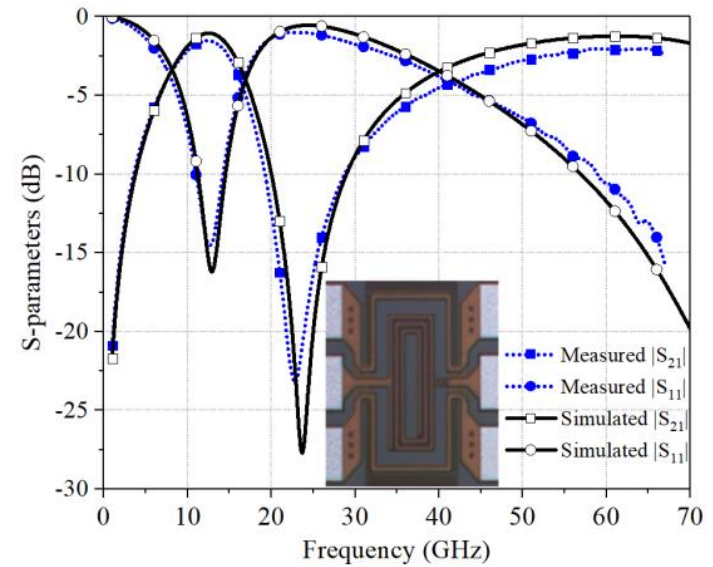

(a)

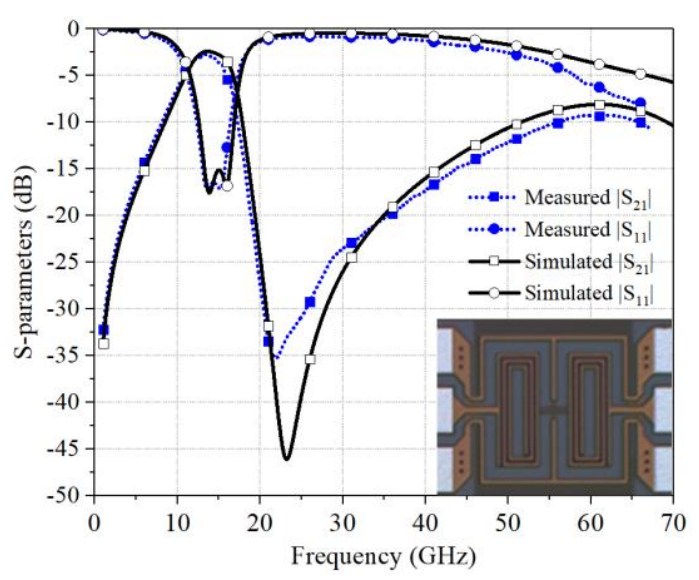

(b)

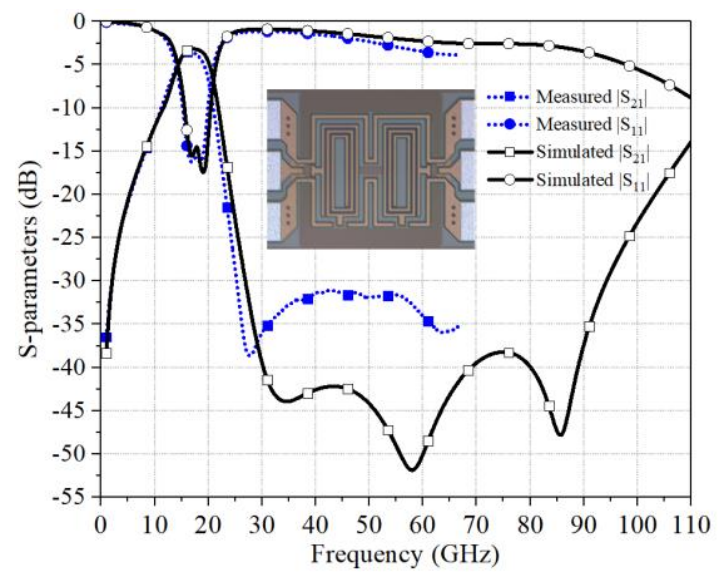

(c)

Fig. 21. Measured S-parameters of all three designs, (a) $1^{\text {st }}$-order BPF, (b) $2^{\text {nd }}-$ order BPF, (c) modified $2^{\text {nd }}$-order BPF with improved out-of-band suppression.

\section{MEAsurement RESUlts AND Discussions}

\section{A. On-Wafer Measurement Results}

To fully evaluate the performance of the above presented designs, all three BPFs are fabricated in a standard $0.13-\mu \mathrm{m}$ (Bi)-CMOS technology. The die microphotographs are embedded with the measured results, which are given in Fig. 20. Excluding the pads, their physical dimensions are $0.13 \times$
$0.25 \mathrm{~mm}^{2}, 0.26 \times 0.25 \mathrm{~mm}^{2}, 0.24 \times 0.22 \mathrm{~mm}^{2}$, respectively. Using a vector network analyser (VNA), ME7838A, from Anritsu, all circuits are measured via on-wafer G-S-G probing, from $1 \mathrm{GHz}$ up to $67 \mathrm{GHz}$. Measurements were made by using conventional open-short-load-through (OSLT) on-wafer calibration to move the reference planes from the connectors of the equipment to the tips of the RF probes. For comparison, both the simulated and measured $\left|S_{21}\right|$ and $\left|S_{11}\right|$ (also known as return loss) of all the three designs are plotted in each figure.

For the $1^{\text {st }}$-order BPF, the results are shown in Fig. 21(a). As can be seen, it has a center frequency at $12.5 \mathrm{GHz}$ with a bandwidth of $24 \%$. The minimum insertion loss is $1.5 \mathrm{~dB}$, while the maximum out-of-band suppression of $23.3 \mathrm{~dB}$ is obtained at $23 \mathrm{GHz}$. The results of the $2^{\text {nd }}$-order BPF that simply cascades two $1^{\text {st }}$-order BPF with some additional inter-stage capacitors are presented in Fig. 21(b). It has a centre frequency of $14 \mathrm{GHz}$ with a $28.6 \%$ bandwidth. The minimum insertion loss and maximum out-of-band suppression are $2.5 \mathrm{~dB}$ and $35 \mathrm{~dB}$, respectively. Finally, the results for the modified $2^{\text {nd }}$-order BPF are given in Fig. 21(c). As can be seen, this design has a center frequency at $17 \mathrm{GHz}$ with a bandwidth of $27.8 \%$. In addition, the minimum insertion loss is $3.5 \mathrm{~dB}$ and the out-of-band suppression is better than $30 \mathrm{~dB}$ from 25 to $67 \mathrm{GHz}$ (limited by equipment).

All in all, fairly reasonable agreements between the EM simulated and the measured results for all three designs have been achieved. The discrepancy between them and some ripples appeared in the tested results below $20 \mathrm{~dB}$ are likely to be caused by the G-S-G pads and testing environment, which are not included in EM simulation.

\section{B. Discussions}

To demonstrate the performance improvement of the presented design over other state-of-the-art ones, a comparison table is given in Table I. Depending on different design specifications, a BPF can be designed with flexibility to satisfy different requirements using the concept presented in this work. Comparing the Design 1 and 2 with other works, they have achieved the lowest insertion loss with miniaturized physical dimensions. Although the insertion loss of the Design 3 is slightly higher than the average one, it has successfully demonstrated a superior out-of-band suppression to the $4^{\text {th }}$-order harmonic frequency at least.

It is noted that some parasitic capacitance from another side of the inductor to the ground is not considered in the equivalent circuit, because it is fairly small comparing with other parasitic capacitance, and thus it only has marginal impact on frequency responses of BPF design. For example, if the parasitic capacitors at Ports 1 and 2 are added in the equivalent circuit, the frequency responses of the BPF will not change much, in terms of the bandwidth, center frequency, positions of transmission zeros, in-band ripple and stopband level. Therefore, the parasitic capacitance is neglected in the synthesis for simplicity. Moreover, although the methodology in this work is only demonstrated for BPFs design around 15 $\mathrm{GHz}$, the method can be directly transferred to higher operation frequencies as well. There is no fundamental limitation for 
TABLE I

PERFormance Summary OF THE DESIGNEd BPFs with OtHer STATE-OF-THE-ART DESIGNS

\begin{tabular}{|c|c|c|c|c|c|c|c|c|c|}
\hline REF. & $\begin{array}{c}f c \\
(\mathrm{GHz})\end{array}$ & $\begin{array}{l}\text { Insertion } \\
\text { loss }(\mathrm{dB})\end{array}$ & $\begin{array}{l}\text { Filter } \\
\text { order }\end{array}$ & $\begin{array}{c}\text { Fractional } \\
\text { bandwidth }(\%)^{\wedge}\end{array}$ & $\begin{array}{l}\text { No. of } \\
\text { TZs }\end{array}$ & $\begin{array}{l}\text { Stopband bandwidth @ more } \\
\text { than } 30 \mathrm{~dB} \text { suppression }(\mathrm{GHz})\end{array}$ & $\begin{array}{l}\text { Stopband suppression } \\
@ \text { high frequency }(\mathrm{dB})\end{array}$ & $\begin{array}{c}\text { Area } \\
\left(\mathrm{mm}^{2}\right)\end{array}$ & Tech. $(\mu \mathrm{m})$ \\
\hline [3] & 60 & 9.3 & $1^{\text {st }}$ & 10 & $\mathrm{n} / \mathrm{a}$ & $\mathrm{n} / \mathrm{a}$ & $\mathrm{n} / \mathrm{a}$ & 0.11 & $0.18 \mathrm{CMOS}$ \\
\hline$[5]$ & 25 & 2.5 & $2^{\text {nd }}$ & 31 & 3 & $51-70$ & 38 & 0.28 & $0.13 \mathrm{CMOS}$ \\
\hline [12] & 65 & 3.4 & $2^{\text {nd }}$ & 18.5 & 2 & $\mathrm{n} / \mathrm{a}$ & 30 & 0.074 & $0.18 \mathrm{CMOS}$ \\
\hline [17] & 60 & 2.5 & $1^{\text {st }}$ & 21 & 2 & $\mathrm{n} / \mathrm{a}$ & 20 & 0.156 & $0.18 \mathrm{CMOS}$ \\
\hline [18] & 59.5 & 3.3 & $2^{\text {nd }}$ & 21.7 & 2 & $\mathrm{n} / \mathrm{a}$ & 27 & 0.054 & $0.18 \mathrm{CMOS}$ \\
\hline [20] & 35 & 4.5 & $2^{\text {nd }}$ & 37.8 & 2 & $56-85$ & 50 & 0.124 & $0.18 \mathrm{CMOS}$ \\
\hline [21] & 33 & 2.6 & $2^{\text {nd }}$ & 42.4 & $\mathrm{n} / \mathrm{a}$ & $72-110$ & $\mathrm{n} / \mathrm{a}$ & 0.03 & $0.13 \mathrm{SiGe}$ \\
\hline [22] & 35 & 3.1 & $3^{\text {rd }}$ & 50 & 3 & $55-62$ & 35 & 0.075 & $0.13 \mathrm{SiGe}$ \\
\hline [23] & 40 & 1.7 & $1^{\text {st }}$ & 20 & 1 & $\mathrm{n} / \mathrm{a}$ & 21 & 0.012 & $0.13 \mathrm{SiGe}$ \\
\hline [24] & 31 & 2.4 & $1^{\mathrm{st}}$ & 22.6 & 1 & $\mathrm{n} / \mathrm{a}$ & 20 & 0.024 & $0.13 \mathrm{SiGe}$ \\
\hline [25] & 33 & 2.6 & $1^{\mathrm{st}}$ & 18 & 1 & $58-70$ & 44 & 0.031 & $0.13 \mathrm{SiGe}$ \\
\hline DESIGN 1 & 12.5 & 1.5 & $1^{\text {st }}$ & 24 & 2 & $\mathbf{n} / \mathbf{a}$ & 23 & 0.033 & 0.13 SiGe \\
\hline DESIGN 2 & 14 & 2.5 & $2^{\text {nd }}$ & 28.6 & 2 & $20-26$ & 35 & 0.065 & 0.13 SiGe \\
\hline DESIGN 3 & 17 & 3.5 & $2^{\text {nd }}$ & 27.8 & 3 & $25-67$ & 38 & 0.053 & 0.13 SiGe \\
\hline
\end{tabular}

using this method to implement a BPF at a higher frequency, such as 30 or $60 \mathrm{GHz}$. A relatively lower frequency is chosen due to the fact that the motivation behind this work is miniaturization. When operation frequency of passive devices is pushing up to millimeter-wave region, their physical dimensions are inherently shrinking. Thus, the miniaturization of devices becomes less critical. Moreover, due to the limitation of the measurement systems, the S-parameters can only be measured up to $67 \mathrm{GHz}$, which means if the BPFs were designed at higher frequencies, it would be quite difficult to measure the frequency responses at the upper-stopband range, especially to investigate the suppression capability of the $2^{\text {nd }}$ and $3^{\text {rd }}$-order harmonics.

\section{CONCLUSIONS}

In this work, two different coupling structures, namely inverting coupling and non-inverting coupling, are analysed and compared. The possibility of using them for miniaturized BPFs design is investigated. To fully understand the insight of these structures as well as effectively use them for BPFs design, simplified $L C$-equivalent circuit models are developed. Using the models, three BPFs are designed and implemented. To further prove that the presented approach is feasible in practice, all designs are fabricated in a standard $0.13-\mu \mathrm{m}$ (Bi)-CMOS technology. The sizes of them without pads are very small. A reasonable agreement between the EM simulated and measured results for all designs is obtained. According to the overall performances of the designed BPFs, it can be concluded that this approach is particularly suitable for miniaturized RFIC design in silicon-based technologies.

\section{REFERENCES}

[1] K. Y. Wu, X. Ding, M. Ismail and H. Olsson, "RF bandpass filter design based on CMOS active inductors," IEEE Trans. Circuits Syst. II, vol. 50, no. 12, pp. 942-949, Dec. 2003.

[2] G. Leuzzi, V. Stornelli, S. D. Re, "A Tuneable Active Inductor With High Dynamic Range for Band-Pass Filter Applications," IEEE Trans. Circuits Syst. II, vol. 58, no. 10, pp. 647-651, 2011.

[3] L. Nan, K. Mouthaan, Y. Z. Xiong, J. Shi, S. C. Rustagi, and B. L. Ooi, "Design of $60-$ and $77-\mathrm{GHz}$ narrow-bandpass filters in CMOS technology,” IEEE Trans. Circuits Syst. II, Exp. Briefs, vol. 55, no. 8, pp. 738-742, Aug. 2008.

[4] J. R. Long, Y. Zhao, W. Wu, M. Spirito, L. Vera, and E. Gordon, "Passive circuit technologies for mm-wave wireless systems on silicon," IEEE Trans. Circuits Syst. I, Reg. Papers, vol. 59, no. 8, pp. 1680-1693, Aug. 2012.

[5] C. - L. Yang, S. -Y. Shu, and Y. -C. Chiang, "Design of a K-band chip filter with three tunable transmission zeros using a standard $0.13-\mu \mathrm{m}$ CMOS technology," IEEE Trans. Circuits Syst. II, Exp. Briefs, vol. 57, no. 7, pp. 522-526, Jul. 2010.

[6] R. Shu, J. Li, T. Adrian, B. J. Drouin, and Q. J. Gu, "Coupling-inductor based hybrid mm-wave CMOS SPST switch," IEEE Trans. Circuits Syst. II, Exp. Briefs, vol. 64, no. 4, pp. 367-371, Apr. 2017.

[7] Z. J. Hou et al., "A W-band balanced power amplifier using broadside coupled strip-line coupler in SiGe Bi-CMOS 0.13- $\mu \mathrm{m}$ technology," IEEE Trans. Circuits Syst. I, Reg. Papers, vol. 65, no. 7, pp. 2139-2150, Jul. 2018.

[8] H. Chung, Q. Ma and G. M. Rebeiz, "A 10-40 GHz frequency quadrupler source with switchable bandpass filters and $>30 \mathrm{dBc}$ harmonic rejection," IEEE Radio Frequency Integrated Circuits Symposium (RFIC), pp. 49-52. Honolulu, HI, 2017.

[9] S. Sun, J. Shi, L. Zhu, S. C. Rustagi, and K. Mouthaan, "Millimeter-wave bandpass filters by standard $0.18-\mu \mathrm{m}$ CMOS technology," IEEE Electron Device Lett., vol. 28, no. 3, pp. 220-222, Mar. 2007.

[10] W. Z. Chen, W. H. Chen, and K. C. Hsu, "Three-dimensional fully symmetric inductors, transformer, and balun in CMOS technology," IEEE Trans. Circuits Syst. I, Reg. Papers, vol. 54, no. 7, pp. 1413-1423, Jul. 2007.

[11] M. K. Chirala, X. Guan and C. Nguyen, "Integrated multilayered on-chip inductors for compact CMOS RFICs and their use in a miniature distributed low-noise-amplifier design for ultra-wideband applications," IEEE Trans. Microw. Theory Tech., vol. 56, no. 8, pp. 1783-1789, Aug. 2008.

[12] S. -C. Chang, Y. -M. Chen, S. -F. Chang, Y. -H. Jeng, C. -L. Wei, C. -H. Huang and C. -P. Jeng, "Compact millimeter-wave CMOS bandpass filters using grounded pedestal stepped-impedance technique," IEEE Trans. Microw. Theory Tech., vol. 58, no. 12, pp. 3850-3859, Dec. 2010.

[13] Y. -S. Lin and V. K. Nguyen, "94-GHz CMOS power amplifiers using miniature dual Y-shaped combiner with RL load," IEEE Trans. Circuits Syst. I, Reg. Papers, vol. 64, no. 6, pp. 1285-1298, Jun. 2017.

[14] I. C. H. Lai, Y. Kambayashi and M. Fujishima, "60-GHz CMOS down-conversion mixer with slow-wave matching transmission lines," Proc. IEEE Asian Solid-State Circuits Conf., pp. 195-198, Nov. 2006.

[15] A.-L. Franc, E. Pistono, G. Meunier, D. Gloria, P. Ferrari, “A lossy circuit model based on physical interpretation for integrated shielded slow-wave CMOS coplanar waveguide structures," IEEE Trans. Microw. Theory Techn., vol. 61, no. 2, pp. 754-763, Feb. 2013. 
[16] K. Kim and C. Nguyen, "An ultra-wideband low-loss millimeter-wave slow-wave Wilkinson power divider on $0.18-\mu \mathrm{m}$ SiGe Bi-CMOS process," IEEE Microw. Wireless Componen. Lett., vol. 25, no. 5, pp. 331-333, May 2015.

[17] N. Mahmoud, A. Barakat, A. B. Abdel-Rahman, A. Allam, R. K. Pokharel, "Compact size on-chip $60 \mathrm{GHz}$ H-shaped resonator BPF," IEEE Microw. Wireless Compon. Lett., vol. 26, no. 9, pp. 681-683, Sep. 2016.

[18] A. S. A. EI-Hameed, A. Barakat, A. B. Abdel-Rahman, A. Allam, R. K. Pokharel, "Ultra compact $60-\mathrm{GHz}$ CMOS BPF employing broadsided-coupled open-loop resonators," IEEE Microw. Wireless Compon. Lett., vol. 27, no. 9, pp. 818-820, Sep. 2017.

[19] P. Sarafis, A. G. Nassiopoulou, H. Issa, and P. Ferrari, "High performance on-chip low-pass filters using CPW and slow-wave-CPW transmission lines on porous silicon," IEEE Trans. Electron Devices, vol. 63, no. 1, pp. 439-445, Jan. 2016.

[20] L. -K. Yeh, C. -Y. Chen and H. -R. Chuang, "A millimeter-wave CPW CMOS on-chip bandpass filter using conductor-backed resonators," IEEE Electron Devices Lett., vol. 31, no. 5, pp. 399-401, May 2010.

[21] Y. Yang, H. Liu, Z. J. Hou, X. Zhu, E. Dutkiewicz, and Q. Xue, "Compact on-chip bandpass filter with improved in-band flatness and stopband attenuation in $0.13-\mu \mathrm{m}$ (Bi)-CMOS technology," IEEE Electron Device Lett., vol. 38, no. 10, pp. 1359-1362, Oct. 2017.

[22] H. Zhu, X. Zhu, Y. Yang and Q. Xue, "Design of wideband third-order bandpass filters using broadside-coupled resonators in $0.13-\mu \mathrm{m}$ (Bi)-CMOS technology," IEEE Trans. Microw. Theory Techn. Early Access.

[23] H. Zhu, Y. Yang, X. Zhu, Y. Sun, and S. W. Wong, "Miniaturized resonator and bandpass filter for silicon-based monolithic microwave and millimeter-wave integrated circuits," IEEE Trans. Circuits Syst. I, Reg. Papers, vol. 65, no. 12, pp. 4062-4071, Dec. 2018.

[24] S. Chakraborty, et al., "A broadside-coupled meander-line resonator in $0.13-\mu \mathrm{m}$ SiGe technology for millimeter-wave application," IEEE Electron Devices Lett., vol. 37, no. 3, pp. 329-331, Mar. 2016.

[25] Y. Zhong, Y. Yang, X. Zhu, E. Dutkiewicz, K. M. Shum and Q. Xue, "An on-chip bandpass filter using a broadside-coupled meander line resonator with a defected-grounded structure," IEEE Electron. Device Lett., vol. 38, no. 5, pp. 626-629, May. 2017.

[26] Y. Yang, X. Zhu, E. Dutkiewicz, and Q. Xue, "Design of a miniaturized on-chip bandpass filter using edge-coupled resonators for millimeterwave applications," IEEE Trans. Electron Devices, vol. 64, no. 9, pp. 3822-3828, Sep. 2017.

[27] J. S. Hong and M. J. Lancaster, Microstrip Filter for RF/Microwave Applications. Hoboken, NJ, USA: Wiley, 2001.

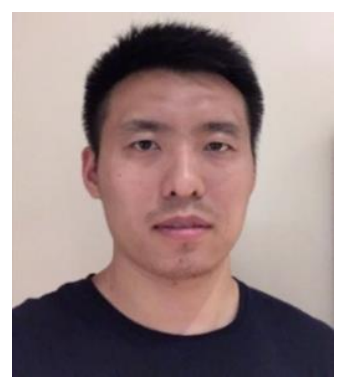

He Zhu (M'18) received the bachelor's degree and master's degrees from South China University of Technology (SCUT), Guangzhou, China, in 2011 and 2014, and the Ph.D. degree from School of Information Technology and Electrical Engineering (ITEE), The University of Queensland, Brisbane,

Australia, in 2017.

He is currently a Post-doctoral Research Fellow with Global Big Data Technologies Centre (GBDTC), University of Technology Sydney, Ultimo, NSW, Australia. His research interests include the development of tunable microwave and mm-wave devices, radio frequency integrated circuits and systems, and beam-forming networks for antenna arrays.

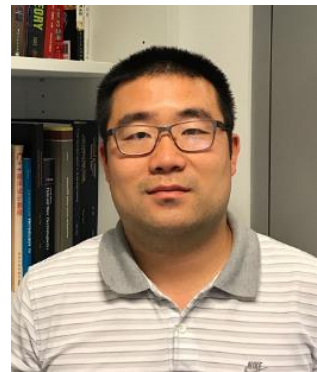

Xi Zhu (M'18) received the B.E. (Hons.) and $\mathrm{PhD}$ from University of Hertfordshire (UH), Hertfordshire, UK, in 2005 and 2008, respectively. He is currently a Lecturer with the School of Electrical and Data Engineering, Faculty of Engineering \& IT, University of Technology Sydney, NSW, Australia. His research activities mainly involve in the areas of analogue baseband, radio frequency (RF) and mm-wave circuits and systems design. He has co-authored over 80 refereed publications in the above-mentioned fields.

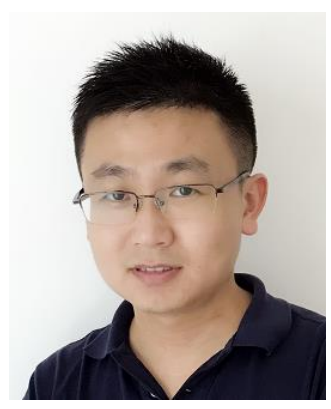

Yang Yang (S'11-M'14-SM'17) was born in Bayan Nur, Inner Mongolia, China and received the $\mathrm{PhD}$ degree from Monash University, Melbourne, Australia, in 2013.

From July 2012 to April 2015, he was an Asia Pacific GSP Engineer at Rain Bird and a Global GSP Success Award holder of the year 2014. From April 2015 to April 2016, he served as a Senior Research Associate with Department of Engineering, Macquarie University, Sydney, Australia. From April 2016 to December 2016, he was a Research Fellow with State Key Laboratory of Millimeter-Waves, City University of Hong Kong. Dr. Yang is currently a Senior Lecturer of University of Technology Sydney, Australia. His research interests focus on millimeter-wave circuits and antennas for $5 \mathrm{G}$ and beyond, and wearable medical sensing devices. He is a current Associate Editor of IEEE ACCESS and a current Area Editor of MICROWAVE OPTICAL TECHNOLOGY LETTERS.

Dr Yang is a winner of CST University Publication Award 2018, by CST, Germany.

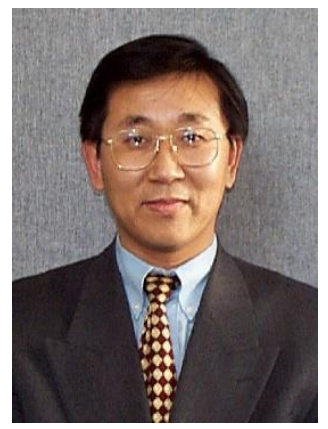

Yichuang Sun (M'90-SM'99) received the B.Sc. and M.Sc. degrees from Dalian Maritime University, Dalian, China, in 1982 and 1985, respectively, and the Ph.D. degree from the University of York, York, U.K., in 1996, all in communications and electronics engineering.

Dr. Sun is currently Professor of Communications and Electronics, Head of Communications and Intelligent Systems Research Group, and Head of Electronic, Communication and Electrical Engineering Division in the School of Engineering and Computer Science of the University of Hertfordshire, UK. He has published over 330 papers and contributed 10 chapters in edited books. He has also published four text and research books: Continuous-Time Active Filter Design (CRC Press, USA, 1999), Design of High Frequency 
Integrated Analogue Filters (IEE Press, UK, 2002), Wireless Communication Circuits and Systems (IET Press, 2004), and Test and Diagnosis of Analogue, Mixed-signal and RF Integrated Circuits - the Systems on Chip Approach (IET Press, 2008). His research interests are in the areas of wireless and mobile communications, RF and analogue circuits, microelectronic devices and systems, and machine learning and deep learning.

Professor Sun was a Series Editor of IEE Circuits, Devices and Systems Book Series (2003-2008). He has been Associate Editor of IEEE Transactions on Circuits and Systems I: Regular Papers (2010-2011, 2016-2017, 2018-2019). He is also Editor of ETRI Journal, Journal of Semiconductors, and Journal of Sensor and Actuator Networks. He was Guest Editor of eight IEEE and IEE/IET journal special issues: High-frequency Integrated Analogue Filters in IEE Proc. Circuits, Devices and Systems (2000), RF Circuits and Systems for Wireless Communications in IEE Proc. Circuits, Devices and Systems (2002), Analogue and Mixed-Signal Test for Systems on Chip in IEE Proc. Circuits, Devices and Systems (2004), MIMO Wireless and Mobile Communications in IEE Proc. Communications (2006), Advanced Signal Processing for Wireless and Mobile Communications in IET Signal Processing (2009), Cooperative Wireless and Mobile Communications in IET Communications (2013), Software-Defined Radio Transceivers and Circuits for 5G Wireless Communications in IEEE Transactions on Circuits and Systems-II (2016), and the 2016 IEEE International Symposium on Circuits and Systems in IEEE Transactions on Circuits and Systems-I (2016). He has also been widely involved in various IEEE technical committee and international conference activities. 